Crimped collagen

Adv. Mater. doi:10.1002/adma.200903612 (2010)

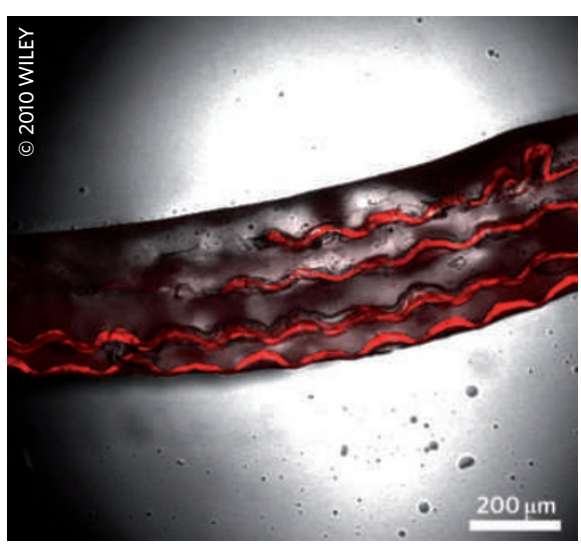

In human tissue, collagen fibres are often observed to form crimped or wavy microstructures. The reproduction of this crimped morphology in synthetic scaffolds may enhance the mechanical behaviour and cellular responses of engineered tissues. Now, Elliot Chaikof and colleagues have developed a way to make collagen fibreelastin composites in which the embedded fibres have a repetitive crimp pattern with a periodicity similar to that of native collagen fibres. First, a parallel array of collagen fibres is sandwiched between a polyurethane substrate and a ridged polyurethane membrane. The substrate and membrane are both pre-extended, and when they relax, the crimp pattern forms in the fibres. The pattern is preserved by crosslinking, and then the fibres are embedded in an elastinlike protein matrix. Subsequently, these collagen fibre-elastin sheets are stacked to form multilamellar composites (pictured) that show similar mechanical behaviour to native tissue. The orientation of fibres in successive layers of the multilamellar material and the presence of either straight or crimped fibres were both shown to alter the mechanical properties of the composites.

\section{Polymer detectives \\ Angew. Chem. Int. Ed. doi:10.1002/ \\ anie.200906720 (2010)}

Ion-selective electrodes (ISEs) with polymeric membranes are commonly used to detect ionic species in clinical and environmental applications. However, ISEs that sense neutral molecules are rare. Rong-Ning Liang et al. have produced an ISE for the potentiometric sensing of the neutral pesticide chlopyrifos (CPF) based on specific molecular recognition of the analyte and an indicator ion. Molecularly imprinted polymers (MIPs) are incorporated into a poly(vinyl chloride) membrane ISE, and an ion with a similar molecular structure to $\mathrm{CPF}$ is used for potentiometric measurements. MIPs are polymers formed around a template, in this case template $\mathrm{CPF}$, which is removed to leave a complementary cavity. Using precipitation polymerization Liang et al. created uniform MIPs, overcoming problems with bulk MIP synthesis and improving sensor response. CPF accumulates in the MIPs when the membranes are immersed in CPF solutions. Potentiometric sensing is then performed by placing these membranes in a solution containing the indicator ions. The response relates to the amount of $\mathrm{CPF}$ accumulated in the membrane and the authors achieved a detection limit for CPF two orders of magnitude smaller than reported previously.

\section{Slippery slope}

Appl. Phys. Lett. 96, 053308 (2010).

With the progressive miniaturization of organic electronic devices, increasingly large electrical fields across thin structures are becoming important for practical

\title{
Surface control
}

The response of cells to physical controls is a topic under a great deal of investigation at present - substrate properties such as texture, geometry and compliance have all been shown to affect cellular responses, although the mechanisms are less clear. Jay Groves and colleagues use engineered membrane surfaces to study how spatial manipulation of receptors on the cells' surface can affect signal-transduction pathways. They use a substrate-supported membrane that contains a set number of ligands that can attach to receptors on the cells' surface; once attached, the receptors are stimulated by the cells to move towards the centre of the cells' underside. The researchers pattern the membrane, inserting metal lines as barriers to the ligands' migration, which in turn arrests the migration of the attached cell receptors. Using this system, Groves and colleagues show that spatially controlling the receptor EphA2, which is over-expressed in $40 \%$ of breast cancer cells, changes cellular responses such as the shape adopted by the cytoskeleton. The results could provide insights into how cell environments contribute to the onset and progression of cancer.

applications. However, the details of the nonlinear high-field transport of charge carriers are often difficult to interpret. Doug Natelson and colleagues have studied the high-field electrical transport in organic semiconductors across a broad range of temperatures. At high temperatures they observe a well-understood thermally activated carrier-hopping transport mechanism. At low temperatures, transport behaviour changes, and carrier transport is largely independent of temperature. To interpret their results, the authors first assumed a one-dimensional Tomonaga-Luttinger liquid model that has recently been evoked to interpret related data in similar samples. However, as is argued, transport measurements across a wide temperature range alone cannot provide unambiguous support for such transport models. Instead, the authors suggest their data follows a temperature-independent transport model based on an emission process of carriers induced by the high applied electric fields. Irrespective of such contradictory conclusions, their study certainly underlines the rich physics occurring in the highfield transport of carriers in organic semiconductors, for which an understanding ultimately requires comprehensive experimental study.

\section{As big as you like}

Nano Lett. doi:10.1021/n19026605 (2010)

That the electronic properties of graphene systems depend strongly on whether there is just one, two or more layers is a wellknown fact. It has now been demonstrated that the number of layers in a graphene film also determines the size of metal particles grown on it. Zhengtang Luo and colleagues examined gold nanoparticles grown by physical vapour deposition on a few-layer graphene substrate, and found that their size is proportional to the cubic route of the number of layers. The data were very well explained by a model based on the electrostatic interaction arising from charge transfer between the graphene and the gold, and that varies with the number of layers, in particular limiting the growth of large particles in the case of a very small number of layers in the substrate. The results suggest the possibility of using few-layer graphene samples for the synthesis of metal nanoparticles with welldefined size for use in various types of application.
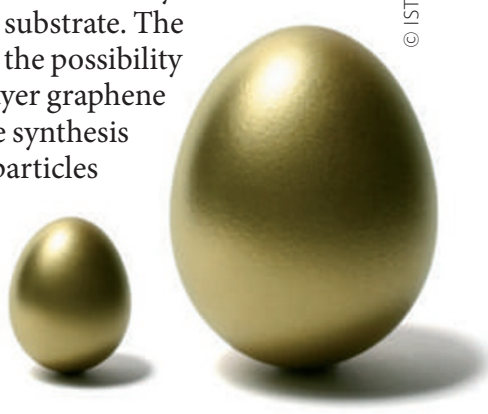